\title{
PROJETO DE IRRIGAÇÃO INTELIGENTE
}

\section{Francisco de Assis Martins dos Santos}

Núcleo de Estudos em Informática no Agreste Potiguar, Instituto Federal de Educação, Ciência e Tecnologia do Rio Grande do Norte, Campus João Câmara, Institucional. Estudante do Curso Subsequente em Informática - IFRN.

E-mail: repitle@ig.com.br

\section{RESUMO}

Neste trabalho é apresentado um método adaptativo para controlar automaticamente um sistema de irrigação. As medições, dos parâmetros climáticos, realizadas pelos sensores são enviados ao microcontrolador que são utilizados como referência pela malha de controle. Essa será responsável por controlar o acionamento do motobomba e dessa forma controlar a lâmina de irrigação.

PALAVRAS-CHAVES: controle, sensor, relé, microcontrolador.

\section{INTELLIGENT IRRIGATION PROJECT}

\begin{abstract}
This paper presents an adaptive method to automatically control an irrigation system. Measurements of climatic parameters, carried out by the sensors are sent to the microcontroller which are used as reference by the control loop. This will be responsible for controlling the actively of the pump and thereby control the water depth.
\end{abstract}

KEY WORDS: control, sensor, relay, microcontroller. 


\section{PROJETO DE IRRIGAÇÃO INTELIGENTE}

\section{INTRODUÇÃO}

O Território do Mato Grande é constituído por 15 municípios: Bento fernandes, Jandaíra, João Câmara, Maxaranguape, Parazinho, Pedra Grande, Poço Branco, Pureza, Rio do Fogo, São Miguel do Gostoso, Taipu, Touros, Caiçara do Norte, São Bento do Norte e CearáMirim. A dinâmica econômica do território é atrelada à produção agropecuária e agricultura [PDS, 2006].

A comercialização da produção era um grave problema enfrentado pela maioria dos agricultores e agricultoras familiares do território, são poucas as alternativas que se tem, praticamente toda a produção é escoada por atravessadores, que normalmente ficam com a maior parte do lucro. A publicação da Lei Federal 11.947 que trata da aquisição de produtos para a alimentação escolar foi bem recebida pelos agricultores. Pois, ficou definido que pelo menos 30\% dos produtos adquiridos para alimentar os estudantes com recursos do Fundo Nacional de Desenvolvimento da Educação (FNDE) devem vir da agricultura familiar. A compra também fica dispensada de licitação, desde que os preços sejam compatíveis com os do mercado local e os produtos atendam normas de qualidade. Resolvendo, assim, um dos principais problemas da agricultura familiar que era a dificuldade de comercialização. Porém, outro problema ainda continua que é a baixa produtividade.

Segundo [Santos et al., 2004] a baixa produtividade média brasileira tem como principal causa a falta de aplicação de práticas culturais adequadas, como espaçamento, época de plantio e consorciação por parte dos produtores. Já a produtividade de culturas agrícolas no Nordeste brasileiro é ainda menor que a média nacional, o que se deve, em parte, ao baixo nível tecnológico empregado por grande parte dos agricultores, pois acreditam que a planta não necessita de muitos cuidados. A disponibilidade hídrica nos primeiros estágios de crescimento da cultura é decisiva no estabelecimento e no aumento da produtividade. Portanto, as lâminas de irrigação influenciam fortemente a cultura plantada [Embrapa, 1999] e consequentemente a produtividade. Então, baseado no exposto verifica-se a é necessidade de implementar técnicas para controlar o processo de irrigação de modo a obter o controle preciso da lâmina de irrigação.

Por isso a vantagem de se implantar nas culturas um sistema de irrigação inteligente. Inteligente, por que o equipamento utilizado vai realizar medições climáticas através de sensores de temperatura, umidade, nível e os que si fizerem necessários de acordo com o tipo de cultura. As referências captadas pelos sensores são enviadas ao microcontrolador que por sua vez será utilizado pela malha de controle que de acordo com os sinais recebidos irá controlar o funcionamento adequado do sistema de irrigação. Por exemplo, imagine que o microcontrolador está simplesmente programado para ligar uma motobomba, caso a temperatura ambiente seja superior a $35^{\circ} \mathrm{C}$ e não esteja chovendo. Nesse caso será necessário utilizar os sensores de temperatura e de chuva.

\section{SENSORES ELETRÔNICOS}

Sensores são dispositivos que mudam seu comportamento sob a ação de uma grandeza física, podendo fornecer diretamente ou indiretamente um sinal que indica esta grandeza. Quando operam diretamente, convertendo uma forma de energia neutra, são chamados transdutores. 
Os de operação indireta alteram suas propriedades, como a resistência, a capacitância ou a indutância, sob ação de uma grandeza, de forma mais ou menos proporcional.

O sinal de um sensor pode ser usado para detectar e corrigir desvios em sistemas de controle, e nos instrumentos de medição, que frequentemente estão associados aos sistemas de controle de malha aberta (não automáticos), orientando o processo.

Os sensores eletrônicos podem ser projetados para detectar uma variedade de aspectos quantitativos de um determinado sistema físico. Tais quantidades incluem: temperaturas, luz (Optoeletrônica), campos Magnéticos, tensão, pressão, deslocamento e rotação, aceleração.

As figuras (1) e (2) mostram os esquemas dos sensores e relé encontrados no Kit PICGenios e utilizados para o desenvolvimento do projeto de irrigação inteligente.

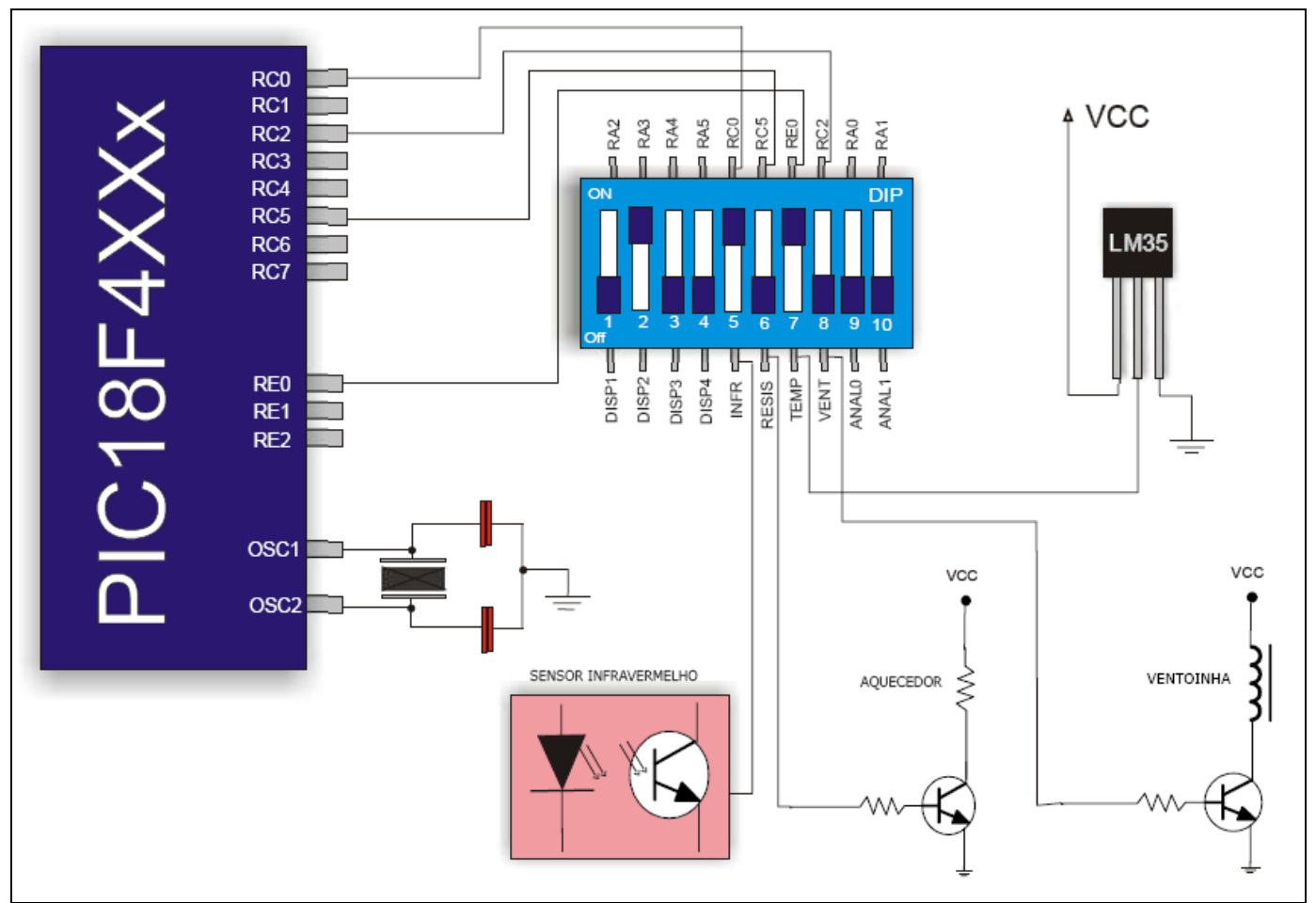

Figura 1 - esquema do PIC $18 f 452$ ligado ao sensor de temperatura $\operatorname{lm} 35$, sensor infravermelho, aquecedor e ventoinha.

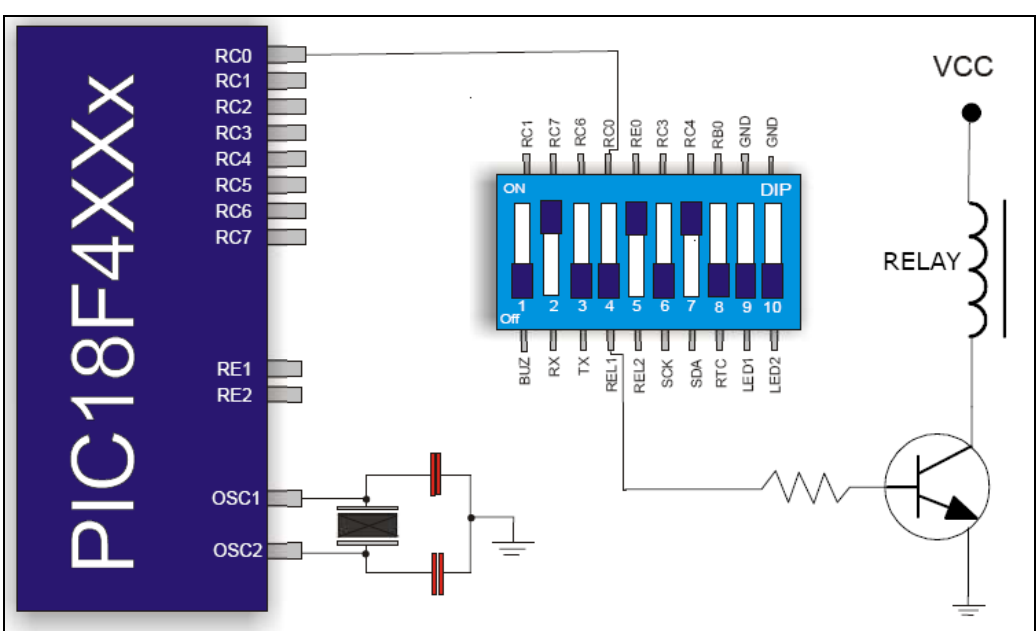

Figura 2 - esquema do PIC $18 f 452$ ligado ao relé, que é ativado de acordo com a variação dos sensores. 


\section{MICROCONTROLADOR PIC18F452}

Um microcontrolador é um circuito integrado que possui internamente um microprocessador e todos os periféricos (qualquer dispositivos que recebe ou transmite os dados ) essenciais ao seu funcionamento, como:

- Memória de programa - geralmente uma memória do tipo EPROM (memória apagável e somente de leitura), onde serão armazenadas as informações de programa, ou seja, o que o microprocessador deve executar;

- Memória de dados - geralmente uma memória do tipo RAM (memória de acesso aleatório), onde ficarão armazenadas as informações de dados que o programa ira utilizar, geralmente é utilizada para guardar um valor;

Cada microcontrolador suporta uma linguagem de programação. A linguagem utilizada nesse trabalho foi C. Todo programador deve conhecer o Set de Instruções de seu microcontrolador para poder realizar a programação do chip.

O código fonte do programa, ou seja, a escrita do programa precisa passar por um compilador, que irá traduzir cada linha digitada em linguagem de máquina, para que o programa possa ser gravado no microcontrolador. A gravação requer muitas vezes um equipamento de gravação. No mercado existem gravadores dedicados e universais que permitem a gravação de inúmeros chips.

O PIC 18F452 mostrado na figura (3) é um modelo de microcontrolador PIC da família de 16 bits de núcleo de processamento, apesar de ainda lidar com dados de 8 bits. A vantagem dessa família de PICs é que eles possuem mais instruções em código de máquina (75 contra 35 da série $16 \mathrm{~F}$ ) que é otimizada para ser usada com compiladores de linguagem C. A sua velocidade de processamento também é maior, na maioria até 10 MIPS (a $40 \mathrm{MHz}$ de clock) com alimentação entre 2 e 5,5V. O seu encapsulamento DIP consiste em 40 pinos. Esse modelo possui $32 \mathrm{k}$ bytes (16k instruções) de memória de programa flash, 1536 bytes de memória RAM e 256 bytes de memória eeprom. Os principais periféricos internos são:

- 8 canais para conversão A/D a 10 bits

- AUSART (addressable universal synchronous assynchronous receiver transmitter) com suporte para RS485

- MSSP (master synchronous serial port) para SPI e I2C

- PSP (parallel slave port)

- 4 timers ( 3 de 16 bits e 1 de 8 bits)

- 2 módulos CCP (capture compare PWM)

- O 18F452 é o modelo de uso geral da família 18F de microcontroladores PIC. 


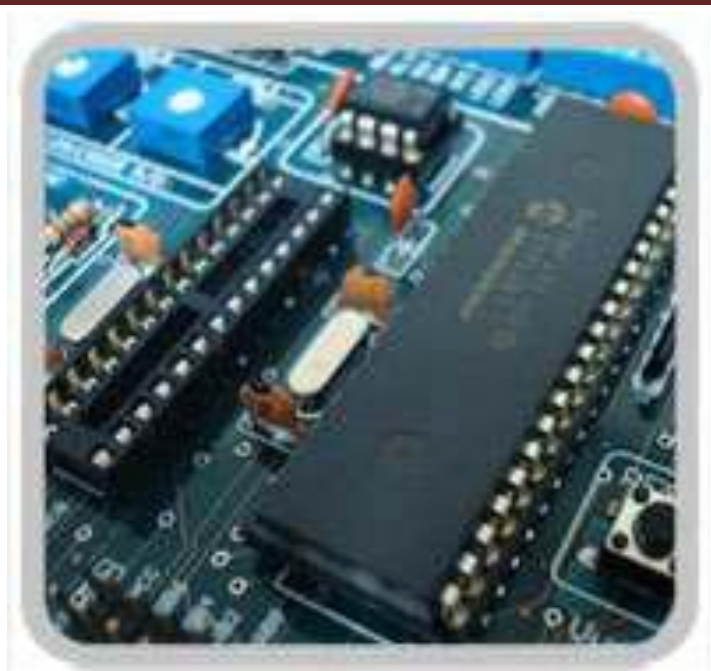

Figura 3 - PIC $18 F 452$

\section{HARDWARE}

Kit PICGenios mostrado na figura (4) acompanha o PIC18F452 que possui 32K FLASH e também suporta PIC18F4550 que possui diversas ferramentas e excelentes recursos disponível, tais como: LCD, USB, PS/2, RS232, RS485, Conversores A/D, Teclado Matricial, RTC (Relógio de Tempo Real), Acionamento PWM, Sensor de Temperatura, Acionamento de Reles, entre outras.

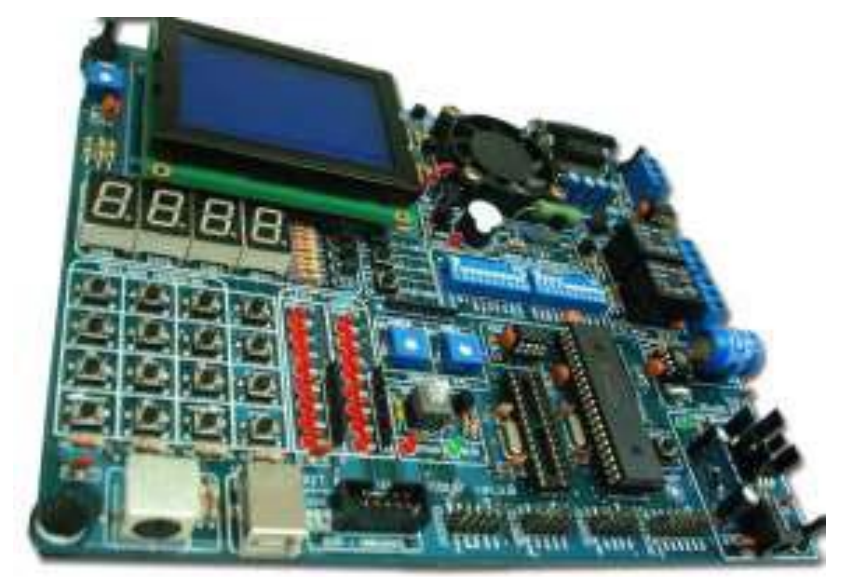

Figura 4 - Kit PICGenios

\section{SOFTWARE}

O PIC18F452 é gravado com instruções em linguagem hexadecimal. A figura (5) mostra a interface do compilador mikroC for PIC, softwares que transforma códigos de linguagem $\mathrm{C} / \mathrm{C}++$ (linguagem de alto nível), para os códigos hexadecimais das instruções do programa a serem gravados no seu microcontrolador PIC. 


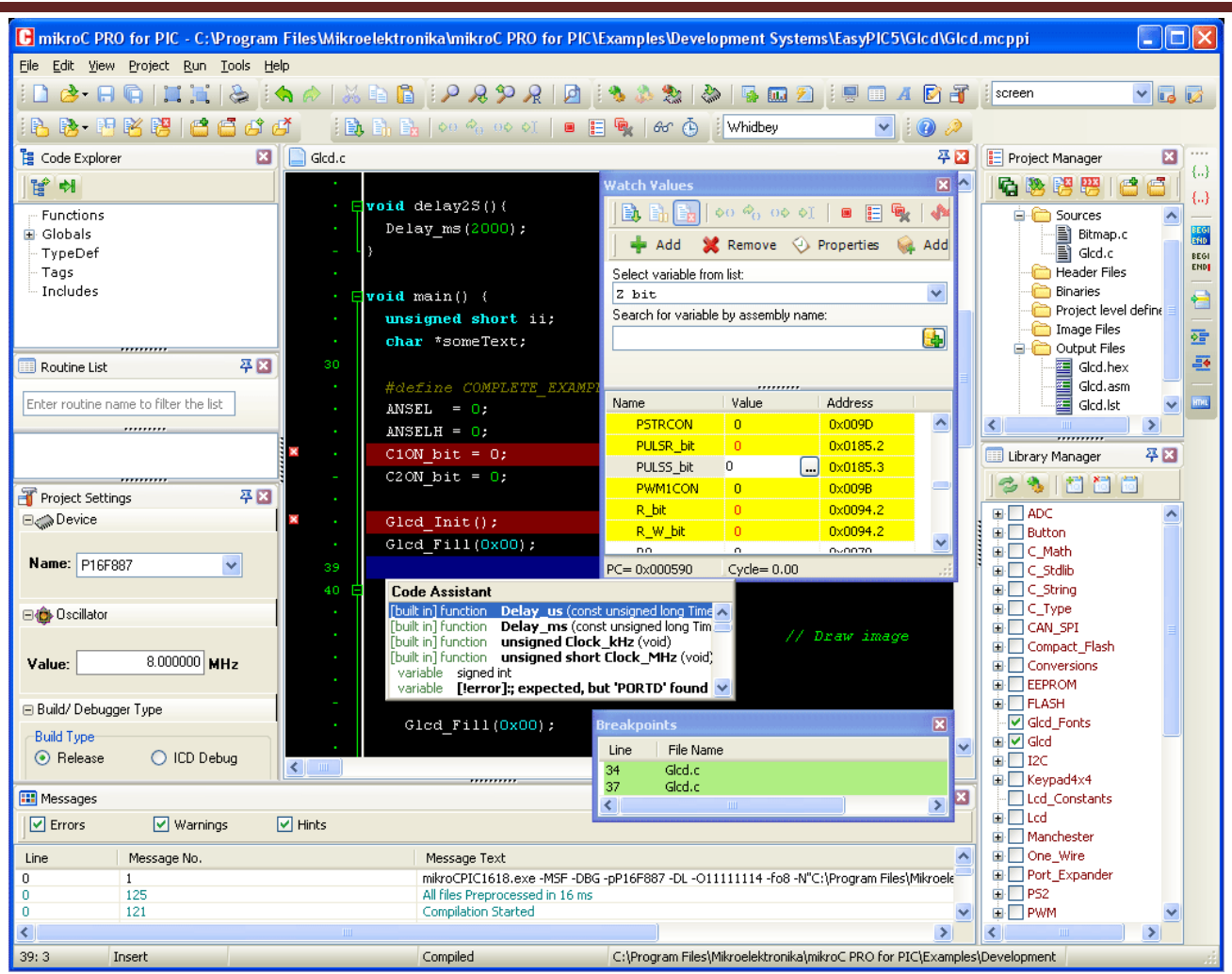

Figura 5 - mikroC for PIC

O software usado está na versão 7.0.0.3 e pode ser executado no Windows® 98/2000/NT/XP/2003/Vista/7.

A figura (6) mostra a interface WinPic800, um programa gratuito para gravação de PICs compatível com vários programadores (hardware) populares disponíveis gratuitamente na internet, e pode ser utilizado na gravação de vários modelos de microcontrolador PIC disponível.

O software está disponível em diversos idiomas incluindo português do Brasil, o software mesmo sendo gratuito sempre terá atualização, para melhor atender a demanda. A interface de comunicação entre esse software e o hardware pode ser realiza através de cabo serial e USB.

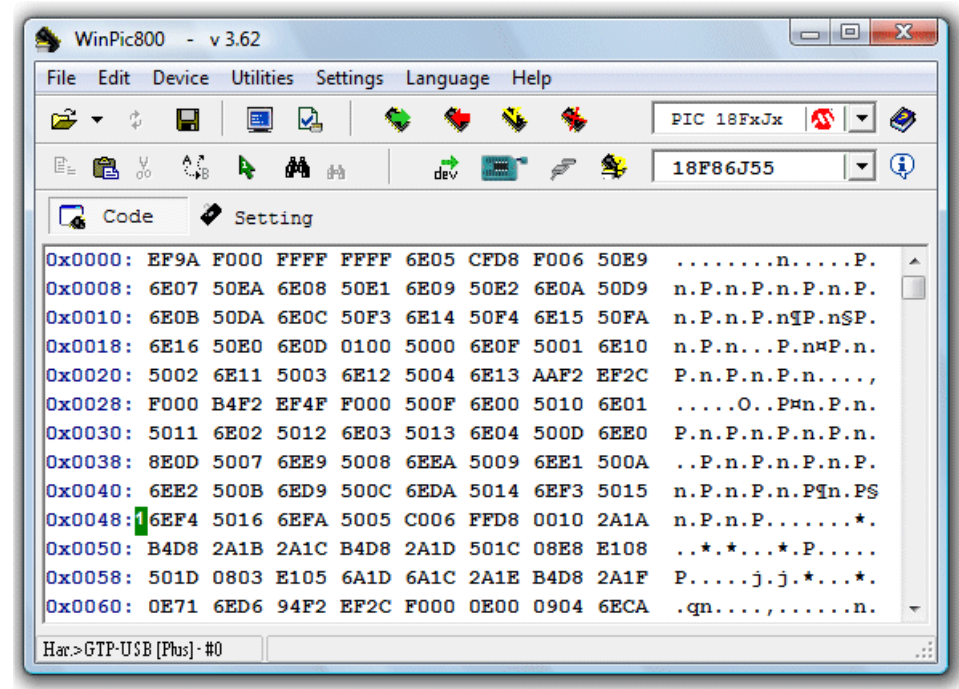

Figura 6 - WinPic800 


\section{PROGRAMAÇÃO}

Para programar o PIC18F452 são necessários:

- Microcontrolador PIC18F452;

- Fonte CC para alimentação do microcontrolador;

- Compilador;

- Microcomputador;

- Sistema Operacional Win98 ou superior;

- Cabo de gravação;

O PIC18F452 utiliza a comunicação serial do PC para gravação, e esta acontece na própria placa do Kit PICGenios.

\section{MATERIAL USADO}

Este projeto foi desenvolvido na Sala de Pesquisa e extensão do Instituto Federal de Educação, Ciência e Tecnologia do Rio Grande do Norte, Campus João Câmara. Foi utilizado um computador HP, AMD Athlon-X2 64 bits, 2GB de Memória RAM. Os equipamentos usados no projeto, além do computador foram:

- Microcontrolador PIC18F452;

- Placa do Kit PICGenios;

- Display LCD serial;

- Sistema Operacional WinXP-SP3;

- Cabo serial de gravação;

\section{SISTEMA DE CONTROLE}

O sistema de controle é um ponto crucial para o funcionamento correto do sistema proposto, pois é a definição da técnica de controle a ser utilizada que garanta estabilidade, robustez, uma resposta dinâmica rápida e erro nulo em regime permanente. O controlador utilizado no sistema de irrigação inteligente foi o PI (Proporcional integrativo). Na figura 7 é apresentado um diagrama de blocos do sistema de controle em malha fechada composto por um controlador PI e a planta (sistema a ser controlado). 


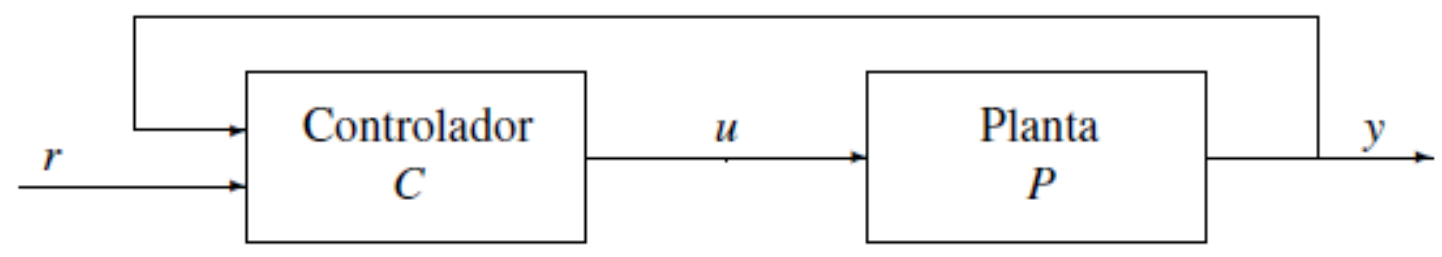

Figura 7 - Diagrama de blocos do sistema de controle

\section{FUNCIONAMENTO E TESTES}

Para testar o sensor de temperatura, foi desenvolvido um código em linguagem $\mathrm{C}$, que depois de compilado e gravado, ativa o aquecedor elevando sua temperatura, o sensor LM35 faz a medição e o resultado dessa medição é mostrado no Display de LCD da placa. Quando a temperatura atinge o nível configurado no microcontrolador o mesmo utilizando o relé, aciona um motobomba iniciando assim a o processo de irrigação por um tempo pré-determinado. Se a temperatura medida for abaixa da referência, como por exemplo, em um dia de chuva, o PIC então não acionará a motobomba. Lembrando que antes da implementação deveram ser analisadas as condições climáticas do local, no intuito de configurar adequadamente o sistema de medição.

\section{CONCLUSÃO}

O desenvolvimento deste projeto demandou muito tempo com o estudo detalhado da linguagem de programação $\mathrm{C}++$, estudo e teste de todos os componentes do microcontrolador PIC18F452. Após, o domínio da linguagem a ser utilizada e de todos os componentes do microcontrolador foi desenvolvido o sistema proposto.

A utilização do sensor de temperatura (LM35) alcançou o resultado esperado para o controle automático, sendo bem sucedido nos testes com o aquecedor, incluso na placa do Kit PICGenios, que foi utilizado para aferir o sistema de medição.

O teste de todo o sistema de irrigação inteligente foi realizado com auxílio do Proteus, buscou-se uma representação realista dos principais componentes do sistema, em que se pode constatar a eficiência do sistema proposto. Porém, a plataforma experimental não foi concluída devida o atraso na compra de alguns dispositivos do projeto.

\section{REFERÊNCIAS BIBLIOGRÁFICAS}

1. PDS, Plano de Desenvolvimento Sustentável da Região do Mato-Grande, 2006.

2. Texas - Apresenta inúmeros applications notes. Encontrado em: www.ti.com

3. Ioannou,P.A.\&J.Sun(1996), Robust Adaptive Control, $2^{a}$ edição, Prentice Hall, San Francisco, EUA.

4. Santos, F. J. de S.; Lima, R. N.; Rodrigues, B. H. N.; Crisostomo, L. A.; Sousa, F. de; Oliveira, J. J. G. Manejo da Irrigação da melancia: Uso do tanque classe "A". Fortaleza: Embrapa Agroindústria Tropical: 2004. 13 p. (Circular Técnica, 20);

5. Empresa Brasileira de Pesquisa Agropecuária - Embrapa. Centro Nacional de Pesquisa de Solo. Sistema de classificação de solos. Brasília, Embrapa: produção de informação, 1999. 